\title{
Exploring embodied haptic design in XR from the epistemology of the Santiago school
}

\author{
Keywords \\ Embodiment, Enaction, Haptic Design, Mixed Reality (XR), Santiago School of Cognition
}

Mixed Reality (MR, and known also as XR) refers to the fusing of real and virtual worlds to create integrated environments that incorporate physical and digital elements, tools, and objects. These environments can be especially powerful within learning contexts as they can assist learners to partake in genuine investigations in the real world. They also allow for the creation of immersive hybrid environments, in which virtual and real objects and experiences are combined. Although immersive digital technologies provide many unique and powerful affordances within $X R$, the role of physical non-digital haptic elements within these contexts has often been dismissed. This is despite the non-digital and haptic showing vast potential for complementing and enhancing the meaningfulness of XR educational experiences. In this context, emerging research is beginning to establish how the biological concepts of autopoiesis, embodiment and enaction from the Santiago School of Cognition, can enhance embodied learning processes within XR education. Embedding principles from the epistemology of the Santiago school within the design of XR experiences encourages learners to interact with, while 'becoming with the world' in a circular experiential motion. Embodied and enactive approaches to designing haptic XR learning experiences can facilitate the creation of tangible, authentic, hands-on and self-determined (i.e. heutagogy) learning experiences with affordances operating as an expansive learning ecosystem. Here we explore how the Santiago school offers a way for educators and learning designers to better develop the haptic and sensory components of XR learning through the concepts of embodiment and enaction. Additionally, embodied cognition in the 'experiencing' process within XR environments can be related to and understood through indigenous worldviews, which are more grounded on a bodily and sensorial experiential relationship with the world. Within the global South, we see a need in bringing together indigenous perspectives, for example Māori cosmologies and worldviews in the case of Aotearoa New Zealand, with southern epistemologies such as the Santiago school. This is because from a western point of view, indigenous concepts can be hard to visualise or ground, but the Santiago school can potentially offer a bridge to understanding these indigenous perspectives within multi-cultural contexts such as Aotearoa. There is also a pressing need to establish new understandings of our critical relationships with nature in the anthropocene, and indigenous perspectives brought forth through the Santiago school can offer this within new forms of learning such as XR. This presentation offers a contribution to the field of $\mathrm{XR}$ education design through the introduction of some novel ways for Art+Science, sensory mapping, and haptic learning design to expand the scope and understanding of this emerging area of educational and practice based research. Engagement with embodied forms of learning connected with indigenous worldviews can allow for deeper connections to be formed between learners and the contexts in which the learning takes place. We postulate here that the notions of embodiment and enaction from the Santiago school are conducive to accessing and bringing forth indigenous cosmologies within XR education. 